\title{
Analysis of Parallel Manufacturing Processes with Resource Sharing
}

\author{
Farooq Ahmad, Hejiao Huang and Xiao-long Wang
}

\begin{abstract}
Multiple resource sharing has a complex nature in parallel manufacturing processes due to the competition of different operations for scarce resources. This paper presents the Petri net model for parallel manufacturing processes with shared resources called parallel process net with resources (PPNR). The structural characteristics of PPNR are discussed and siphon based characterizations of live and reversible PPNR are also presented. Simple structure based conditions and properties for conservative PPNR are presented for checking its liveness and reversibility. Finally, proposed method of net modeling is demonstrated by a practical example.
\end{abstract}

Index Terms-Petri net, resource sharing, parallel processing flows, liveness.

\section{INTRODUCTION}

Handling resource sharing becomes an important aspect during flexible manufacturing system (FMS) design and allocation of resources is always a challenging task in the modeling. Further, the resource-sharing among different operations to be performed does not imply its simultaneous usage because each operation utilizes a resource type exclusively and releases it after completion. Furthermore, a resource type held by one operation can not be preempted by another operation.

The parallel flows of multiple products in a FMS can be identified as different jobs to be completed. For instance, parallel or concurrent processing of raw products of different types on limited number of resources such as machines, robots buffers etc. is a common situation in FMS. While sharing the limited number of resources, these jobs have a particular operation routing that determines the order in which resources must be assigned to the product. Therefore, allocation of limited number of resources to the different operations to achieve the efficient output of FMS is not a trivial task and requires the modeling of operation flows in FMS.

Petri nets (PNs) have been extensively used for modeling, analysis and design of FMS [1]-[6] because of the ability of PNs for formal verification to detect the important behavioral properties of modeled systems. The PN model of a manufacturing system (MS) is either constructed by a top-down or bottom-up approach [7]. In a top-down approach [8]-[10], firstly the high-level description of system is presented and then model is stepwise refined by adding the subnets until a complete net model is achieved. For FMSs with shared resources, the top-down approach has a drawback that the subnets are strongly coupled and it is hard to find the small size of final expanded model. Where as, in bottom-up approach [11]-[13], the net modules of specified subsystems are constructed. Then, they are combined by sharing common places, transitions or subnets. However, the verification of desired set of properties for such type of integrated model is not straightforward. Consequently, the disadvantage of bottom-up approach is the great difficulty in the verification of desired behavior of the large-scale integrated model.

To cope with these shortcomings of modeling approaches, this paper presents a new class of PNs called parallel process net with resources (PPNR) for FMSs having parallel processes. There are two stages which comprise the modeling procedure of PPNR. Firstly, the parallel process net (PPN) is constructed to specify the process flows of each part type in a MS without considering the resources. The step of PPN portraits the parallel processing of parts and depicts the order of different operations. Further, this step assists for the proper assignment of resources required to process each part type and provide the resource-allocation policy according to the given production plan. Thereafter, the marked resource places denoting the availability of resource types are added to the PPN. In this way, PPNR can model more complex resource-sharing and interacting parallel processes in FMSs.

The main power of the PN as mathematical tool is its support for analysis to study important characteristics for synthesis after modeling of the physical MS has been performed. Structural analysis illuminates the important structural characteristics of the PN model and useful for its synthesis. One of the main advantages of the structural approach for characterization of the PN model is that it is independent of the reachable states of a system, which is computationally impractical for large-scale MSs. Moreover, structural approach for the analysis of FMSs is mainly based on minimal siphons [12], [14]-[19]. Although the number of siphons grows quickly and in the worst case grows exponentially fast with respect to the PN size [20], [21], even though it is practical because there is no need to generate the reachability graph which suffers from state-explosion problem [22].

This paper presents a number of characterizations of live and reversible PPNR based on siphons and minimal marked siphons. Further, several structural characteristics of PPN and then PPNR are described. These characteristics help to identify the requirements of the structure of PPNR and its behavior. Furthermore, main interest of the simple structural based conditions and properties, presented in this paper, is in 
their use for the synthesis of live and reversible PPNR.

The paper is organized as follows. Some related terminologies are introduced in Section 2. In Section 3, the formal definition and the characterization of PPN is presented. Section 4 introduces the PPNR and presents the characterization of live and reversible PPNR. The demonstration of the proposed modeling procedure is presented in Section 5 and concluding remarks are presented in Section 6.

\section{DEFINITIONS AND CONCEPTS}

In this section, some basic definitions and notations of ordinary (for the sake of simplicity) PN are described. The related terminology and notations are mostly taken from [23], [24].

Definition 1: (Petri net) A Petri net $P N$, is a five tuple, $P N=\left(P, T, I, O, M_{0}\right)$. Where, $P=\left\{p_{1}, p_{2}, \ldots, p_{|P|}\right\}$ is a finite set of places, $|P|>0 ; T=\left\{t_{1}, t_{2}, \ldots, t_{|T|}\right\}$ is a finite set of transitions, $|T|>0 ; I: T \rightarrow P$ is the input function, which is a mapping from transitions to the set of places and it indicates the input places of transitions; $O: T \rightarrow P$ is the output function, which is a mapping from transitions to the set of places and it indicates the output places of transitions, $P \cap T=\varnothing$ and $P \cup T \neq \varnothing$.

Let $I\left(t_{j}\right)$ represent the set of input places of transition $t_{j} \in T$ and $p_{i} \in P$ is an input place of a transition $t_{j}$ if $p_{i} \in I\left(t_{j}\right) ; O\left(t_{j}\right)$ represents the set of output places, then $p_{i}$ is an output place of $t_{j}$ if $p_{i} \in O\left(t_{j}\right)$.

The input and output functions can be extended to map the set of places $P$ into the set of transitions $T$ such as $I: P \rightarrow T$ and $O: P \rightarrow T$. Then, set $I\left(p_{i}\right)$ represents the set of input transitions of place $p_{i} \in P$ and set $O\left(p_{i}\right)$ represents the set of output transitions of place $p_{i} \in P$.

The incoming arc from $p_{i}$ to $t_{j}$ is represented by $\left(p_{i}, I\left(t_{j}\right)\right)$ and outgoing arc from $t_{j}$ to $p_{i}$ be $\left(p_{i}, O\left(t_{j}\right)\right)$. Similarly, $\left(t_{j}, I\left(p_{i}\right)\right)$ represents the incoming arc from $t_{j}$ to $p_{i}$ as $t_{j} \in I\left(p_{i}\right)$ and $\operatorname{arc}\left(t_{j}, O\left(p_{i}\right)\right)$ represents outgoing arc from $p_{i}$ to $t_{j}$ as $t_{j} \in O\left(p_{i}\right)$, when the set of places maps into the set of transitions; $\forall t_{j} \in T, \forall p_{i} \in P$.

The structure of a PN is defined by the set of places, set of transitions, input function and output function. A PN structure without $M_{0}$ is denoted by $N=(P, T, I, O)$. A PN structure $N$ is said to be strongly connected if and only if every node $x_{i} \in P \cup T$ is reachable from every other node $x_{j} \in P \cup T$ by a directed path. A PN structure $N$ is said to be self-loop-free or pure if and only if $\forall t_{j} \in T$, $I\left(t_{j}\right) \cap O\left(t_{j}\right)=\varnothing$, i.e. no place can be both an input and an output of the same transition. A PN structure $N$ is said to be state-machine if and only if, $\forall t_{j} \in T,\left|I\left(t_{j}\right)\right|=\left|O\left(t_{j}\right)\right|=1$ and said to be marked graph if and only if $\forall p_{i} \in P$, $\left|I\left(p_{i}\right)\right|=\left|O\left(p_{i}\right)\right|=1$.

A marking is a function $M: P \rightarrow \square$ (non-negative integers) and initial marking is denoted by $M_{0}$. A PN with given initial marking is denoted by $\left(N, M_{0}\right)$. The set of all reachable markings from $M_{0}$ is denoted by $R\left(M_{0}\right)$ which is a definite set of markings of $\mathrm{PN}$ such that, if $M_{k} \in R\left(M_{0}\right)$ and $M_{k} \stackrel{t_{j}}{\longrightarrow} M_{k}^{\prime}$ for some $t_{j} \in T$, then $M_{k}^{\prime} \in R\left(M_{0}\right)$.

Definition 2: (Firing rule) The firing rule identifies the transition enabling and the change of marking. Let $M\left(p_{i}\right)$ be the number of tokens in place $p_{i}$, then for $\forall t_{j} \in T ; t_{j}$ is enabled under marking $M$ if and only if $\forall p_{i} \in I\left(t_{j}\right): M\left(p_{i}\right) \geq 1$. The change of marking $M$ to $M^{\prime}$ by firing the enabled transition $t_{j}$ is denoted by $M \stackrel{t_{j}}{\longrightarrow} M^{\prime}$ and defined for each place $p_{i} \in P$ by $M^{\prime}\left(p_{i}\right)=\left\{\begin{array}{l}M\left(p_{i}\right)-1 \text { for every } p_{i} \in I\left(t_{j}\right) \\ M\left(p_{i}\right)+1 \text { for every } p_{i} \in O\left(t_{j}\right) \\ M\left(p_{i}\right) \text { otherwise. }\end{array}\right.$

Definition 3: (P-invariant, T-invariant and conservativeness) For a $\mathrm{PN}\left(N, M_{0}\right)$, a $\mathrm{P}$-invariant is a $|P|$-vector $y \geq 0$ such that $A y=0$, where $A$ is the $|T| \times|P|$ incidence matrix. Similarly, a T-invariant is a $|T|$-vector $x \geq 0$ such that $A^{T} x=0$. A PN is said to be conservative if and only if there exists a P-invariant $y>0$.

Definition 4: (Siphons, traps and minimal siphons) A set of places $S \subseteq P$ is called a siphon if $I(S) \subseteq O(S)$ and it is called a trap if $O(S) \subseteq I(S)$. A siphon $S$ is called minimal if there does not exits $S^{\prime}$ such that $S^{\prime} \subset S$.

Definition 5: (Liveness) A transition $t_{j} \in T$ is said to be live if $\forall M_{k} \in R\left(M_{0}\right)$ there is marking $M_{k}^{\prime}$ reachable from $M_{k}$ such that $M_{k}^{\prime}$ enables $t_{j}$ and $\mathrm{PN}\left(N, M_{0}\right)$ is live if $\forall t_{j} \in T$ : $t_{j}$ is live.

Definition 6: (Reversibility) A marking $M_{k}$ of $\mathrm{PN}$ $\left(N, M_{0}\right)$ is called reversible if and only if for every marking $M_{k}^{\prime}$ reachable from $M_{k}$ there exist a firing sequence reproducing $M_{k}$ and $\left(N, M_{0}\right)$ is called reversible if and only if $M_{0}$ is reversible.

\section{Parallel Process NeT}

The process flow of each part type (raw or in-process material) is represented by the token flow in a PN model. The places are used to model the different operations (e.g., machining, holding, assembling and transformation etc.) to be executed over the part types. The resource types (e.g., machines, buffers, robots, etc.) are also modeled by the initially marked places referring to the availability of resources.

The transitions grant to advance a part type into the finished 
product as they represent the start and termination of manufacturing processes in the PN model. There are output (input) arcs from resource places to those transitions that move a process to (from) the operation state by using these resources. The sequence of transition firing represents the production path in the PN model of a production plan and there may be several paths to achieve a final product in a same plan.

The parallel process net (PPN) is constructed as a first step for the specification of parallel processing flows of each part type in a manufacturing system without considering the resources. The production plan in a manufacturing system has specific input point(s) for raw material and output point(s) for finished product(s). In a PPN, such input points are combined by the single transition with single marked input place as shown in Fig. 1(a). Such single transition, denoted by $t_{0}$, initiates the parallel execution of jobs in a modeled FMS. The single input place of transition $t_{0}$ is called initial place, denoted by $p_{0}$, and every cycle in parallel process net include it. In this way the PPN is strongly connected and self loop free. The output points for finished parallel jobs, which are modeled by places, are synchronized by single transition, called synchronizing transition and denoted by $t_{s}$, as depicted in Fig. 1(a). A token in a place $p_{f}$ in Fig. 1(a), called final place, represents the completion of processing. The transition which finishes the single production iteration is denoted by $t_{f}$ where $p_{f}$ stands for its input place and $p_{0}$ as its output place. For formal definition of PPN, the set of places excluding the initial place $p_{0}$ and final place $p_{f}$ is denoted by $P_{O P}$ and the set of transitions representing the start and termination of different operations in PPN is denoted by $T_{O P}$.

$$
\begin{aligned}
& \text { Definition 7: The PPN is a PN } \\
& P N=\left(P, T, I, O, M_{0}\right) \text { such that } \\
& \text { i. } \quad P=P_{O P} \cup\left\{p_{0}, p_{f}\right\} \\
& \text { ii. } \quad T=T_{O P} \cup\left\{t_{0}, t_{s}, t_{f}\right\} \\
& \text { iii. } \quad I\left(p_{0}\right)=\left\{t_{f}\right\} \text { and } O\left(p_{0}\right)=\left\{t_{0}\right\} \\
& \text { iv. } \quad I\left(t_{0}\right)=\left\{p_{0}\right\} \text { and }\left|O\left(t_{0}\right)\right| \text { is the number of raw parts } \\
& \text { ventering into the system } I\left(p_{f}\right)=\left\{t_{s}\right\} \vee T_{f} \subseteq T_{O P} \text { and } O\left(p_{f}\right)=\left\{t_{f}\right\} \\
& \text { vi. } \quad I\left(t_{f}\right)=\left\{p_{f}\right\} \text { and } O\left(t_{f}\right)=\left\{p_{0}\right\} \\
& \text { vii. } \quad M_{0}\left(p_{0}\right)=1 \Rightarrow M_{0}\left(p_{i}\right)=0 \forall p_{i} \in P \backslash\left\{p_{0}\right\} \\
& \text { viii. } \quad \forall M_{k} \in R\left(M_{0}\right) ; M_{k}\left(p_{f}\right)=1 \Rightarrow M_{k}\left(p_{i}\right)=0 \\
& \quad \forall p_{i} \in P \backslash\left\{p_{f}\right\}
\end{aligned}
$$

Property 1: Every cycle in PPN contains the initial place $p_{0}$, final place $p_{f}$ and transition $t_{f}$.

Proof: The proof for the statement of Property 1 is trivial, as by removing the initial place $p_{0}$ final place $p_{f}$ and transition $t_{f}$ and its associated arcs, PPN is acyclic.

Property 2: Each isolated parallel processing flow in PPN is executable.

Proof: Every processing flow is a sequence of operations represented by the transitions for their beginning and termination, which can be fired if their input places representing the first operation in order are marked. Since $O\left(t_{0}\right)$ are the set of places representing the specific input points for the processing of parts entering into the system, which become marked by firing initial transition $t_{0}$. From (iv) and (vii) of Definition 7, $t_{0}$ is executable.

Property 3: In PPN, an individual parallel processing flow is a T-invariant.

Proof: An independent sequence of operations is for completing the processing of a part entering into the system, which is interpreted as production path. Each cycle in PPN contains such type of production path, which is executable in isolation due to Property 2. From Definition 7(vii), $M_{0}\left(p_{0}\right)=1$ and each cycle contains the initial place $p_{0}$, sequence of transitions in each cycle is a T-invariant.

Property 4: Every cycle in PPN is a strongly connected state-machine.

Proof: Form Property 3, every cycle contains the individual parallel processing of a part represented by the sequence of operations. Further every cycle contains common initial place $p_{0}$, initial transition $t_{0}$, synchronizing transition $t_{s}$, final place $p_{f}$ and final transition $t_{f}$. According to the Property 1, by removing them, there are independent parallel processing flows represented by the sequence of operations, where every transition representing either beginning of an operation or end of operation has only one input and output place representing a specific operation. Hence every cycle is the strongly connected state-machine with the transitions representing the firing sequence that completes the processing of a part entering into the system.

Property 5: Every cycle in PPN is a siphon with initial place $p_{0}$, final place $p_{f}$ and transition $t_{f}$.

Proof: Trivial, corresponding set of places in a cycle is a siphon. From Property 1 , by removing initial place $p_{0}$, final place $p_{f}$ and transition $t_{f}$ there is no cycle in PPN. Hence every cycle is a siphon in PPN containing $p_{0}$, final place $p_{f}$ and transition $t_{f}$.

Property 6: A strongly connected PPN is reversible.

Proof: Every cycle in PPN represents the production routing of parts entering into the system. Further, from Property 3 , every cycle is a T-invariant representing the firing sequence that completes the processing of each part. Therefore, $M_{0}$ is reachable from any intermediate marking $M_{k} \in R\left(M_{0}\right)$ in PPN.

Property 7: A strongly connected PPN is live.

Proof: Property 5 follows that every cycle in PPN is a siphon with common place $p_{0}$, final place $p_{f}$ and transition $t_{f}$. From Properties 2, 3 and 4, every cycle in PPN contains a production path for a part entering into the system and no other cycle exists in it, which follows that every cycle is not only a strongly connected state-machine but also a marked graph. This implies that every siphon in PPN is also a trap. From Definition 7(vii), every cycle is marked, which implies that every siphon has a marked trap. Hence PPN is live due to 
[23].

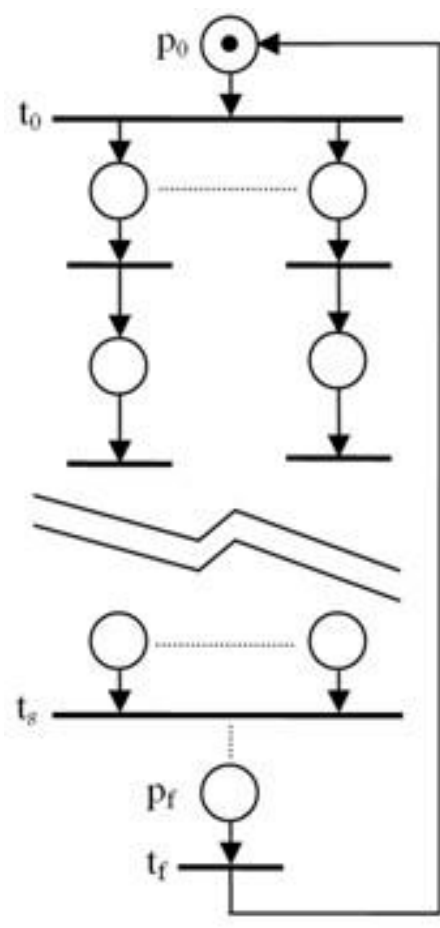

(a)

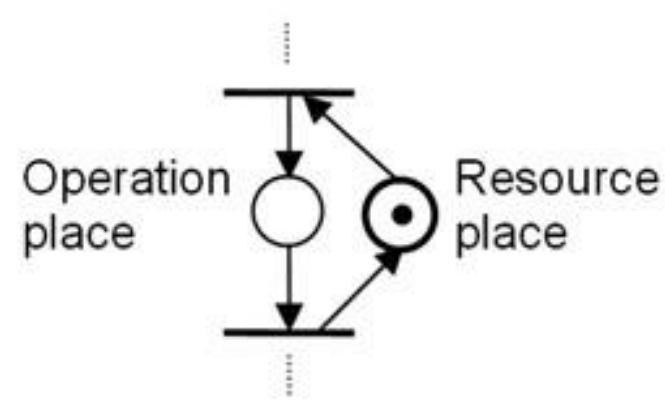

(b)

Fig. 1 (a) The sketch of a parallel process net and (b) assignment of a resource to the process

It is extremely desirable that every transition in PPN models an actual event of process execution or termination and there is not a redundant transition in it. Further, every operation can proceed towards its completion without any interruption and execution of any additional operation. In order to make sure the existence of these requirements, the characterization of PPN illuminates that there is no dead transition in it and it is reversible. Since attention is focused on efficient resource-allocation to the parallel manufacturing processes, the complete characterization of the class of PPN is beyond the scope of this paper.

\section{Parallel Process Net With Resources}

The step of PPN assists for the proper assignment of resources required to process each part type. Thereafter, the marked resource-places denoting the availability of resource types are added to the PPN such that input and output transitions of each operation-place act as its output and input transition respectively, as shown in Fig. 1(b).

The PN model of parallel manufacturing processes with marked resources-places will be called, from now on, parallel process net with resources (PPNR).

For the purpose of defining PPNR formally, the set of places $P$, excluding the initial place $p_{0}$ and final place $p_{f}$, is divided into the set of operation-places $P_{O P}$ and the set of resource-places $P_{R}$.

Definition 8: The PPNR is a PN $P N=\left(P, T, I, O, M_{0}\right)$ such that i. $\quad P=P_{O P} \cup P_{R} \cup\left\{p_{0}, p_{f}\right\}$ and $P_{O P} \cap P_{R}=\varnothing$

ii. $T=T_{O P} \cup\left\{t_{0}, t_{s}, t_{f}\right\}$

iii. $I\left(p_{0}\right)=\left\{t_{f}\right\}$ and $O\left(p_{0}\right)=\left\{t_{0}\right\}$

iv. $I\left(t_{0}\right)=\left\{p_{0}\right\}$ and $O\left(t_{0}\right) \cap P_{R}=\varnothing$

v. $I\left(p_{f}\right)=\left\{t_{s}\right\} \vee T_{f} \subseteq T_{O P}$ and $O\left(p_{f}\right)=\left\{t_{f}\right\}$

vi. $I\left(t_{f}\right)=\left\{p_{f}\right\}$ and $O\left(t_{f}\right)=\left\{p_{0}\right\}$

vii. $M_{0}\left(p_{0}\right)=1 \wedge M_{0}\left(p_{i}\right)=1 \forall p_{i} \in P_{R}$, while

$$
M_{0}\left(p_{f}\right)=0 \wedge M_{0}\left(p_{i}\right)=0 \forall p_{i} \in P_{O P}
$$

viii. $\forall M_{k} \in R\left(M_{0}\right) \quad$;

$$
\begin{aligned}
& M_{k}\left(p_{0}\right)+M_{k}\left(p_{f}\right)=1 \Rightarrow M_{k}\left(p_{i}\right)=0 \\
& \forall p_{i} \in P_{O P}
\end{aligned}
$$

ix. $\quad P N=\left(P \backslash P_{R}, T, I, O, M_{0}\right)$ with $\quad M_{0}\left(p_{0}\right)=1$ and $M_{0}\left(p_{i}\right)=0 \forall p_{i} \in P \backslash P_{R} \cup\left\{p_{0}\right\}$ is a strongly connected and self-loop free PPN.

In the PPNR introduced by Definition 8, a token in place $p_{0}$ represents the instance of waiting to initiate the parallel processing of multiple products while the initiation of a parallel processing is modeled by the firing of transition $t_{0}$. Similarly, token in a place $p_{f}$ represents the completion of processing instances, while the event of a process completion is modeled by the firing of transition $t_{f}$. Transition $t_{f}$ allows re-circulation of token from place $p_{f}$ to place $p_{0}$ in order to model the iterative execution of PPNR to produce the required number of final products.

Property 8: For each $p_{i} \in P_{R}$ there exits a minimal P-invariant with only marked place $p_{i} \in P_{R}$. 
Proof: Since each resource-place $p_{i} \in P_{R}$ is added to the PPN in such a way that input and output transitions of each operation-place $p_{j} \in P_{O P}$ act as its output and input transition respectively, as shown in Fig. 1(b). From Property 4, every individual parallel process is a state-machine. Further,

$I\left(t_{0}\right) \cap P_{R}=O\left(t_{0}\right) \cap P_{R}=I\left(t_{f}\right) \cap P_{R}=O\left(t_{f}\right) \cap P_{R}=\varnothing$.

Therefore, allocation of each resource place to each operation place is represented by directed cycle with only marked place $p_{i} \in P_{R}$ representing the availability of a resource. Hence allocation of resources in PPN imposes the existence of minimal P-invariant for each $p_{i} \in P_{R}$.

Property 8 directly follows that allocation of resources to the operations in PPNR is in conservative way, which implies that resources can neither be created nor destroyed. Moreover,

the existence of P-invariant $y_{i}$ for each resource place $\quad p_{i} \in P_{R} \quad$ follows that $P_{R} \cap\left\|y_{i}\right\|=\left\{p_{i}\right\}$, $P_{O P} \cap\left\|y_{i}\right\| \neq \varnothing$ and $\left\{p_{0}\right\} \cap\left\|y_{i}\right\|=\varnothing$. Property 8 depicts that resources can be iteratively used and released and appropriate for the actual requirement of the manufacturing system.

Property 9: Every minimal P-invariant $y_{i}$ is a minimal siphon with $M_{0}\left(p_{i}\right)=1, \forall p_{i} \in P_{R}$.

Property 9 follows that for each resource place $p_{i}$, there exists a minimal siphon with only marked place $p_{i}$.

Theorem 10: Let $\left(N, M_{0}\right)$ be a PPNR with "non-shared" resources, then it is live.

Proof: Since there is not a dead transition in PPN, it is live. Property 9 implies that every resource added to PPN makes an initially marked minimal siphon with the operation place. Because every operation is performed on an independent resource, therefore every operation place along with a resource place in PPNR also makes a trap, which is marked due to Definition 8(vii). Minimal siphons thus obtained by independent resource places contain a marked trap which directly follows the liveness of PPNR due to [23].

Theorem 10 follows that places representing the shared resources in PPNR have the potential of deadlock because each operation utilizes a resource exclusively and releases it after the completion. For the processing flow of a part, the transition between two operations represents the end of first operation and also the beginning of second operation in order. The utilization of a resource is represented by transferring of a token from resource place to operation place through a transition representing the beginning of an operation on that resource. A transition at any marking of PPNR is said to be enabled if its preceding operation place as well as its input resource place are marked by a token.

Theorem 11: An PPNR is free from deadlocks, if and only if $\nexists M_{k} \in R\left(M_{0}\right)$ such that $M_{k}\left(p_{i}\right)=0$, for any of $p_{i} \in I\left(t_{j}\right)$, for each $t_{j} \in T_{O P}$ representing the beginning of operations at $M_{k}$.

Theorem 11 is related to the non-existence of a marking where the execution of operations is blocked due to the unavailability of resources. The input places of the transitions representing the beginning of operations are unmarked often representing the unavailability of resources. The unavailability of required resource is often due to the reason of holding of that resource by another operation. This situation of circular-waiting leads to the existence of a siphon whose resource places are unmarked.

Theorem 12: An PPNR is live if and only if there does not exists a siphon $S$, such that $\exists M_{k} \in R\left(M_{0}\right)$, $M_{k}\left(p_{i}\right)=0 \forall p_{i} \in P_{R} \cap S$.

Proof: Assume that PPNR is live and that there is siphon $S$ and reachable marking $M_{k}$, for which $M_{k}\left(p_{i}\right)=0 \forall p_{i} \in P_{R} \cap S$. Properties 5 and 9 lead to the fact that every siphon contains an initial place $p_{0}$, a final place $p_{f}$ or at least one resource place. From Theorem 11, $M_{k}\left(p_{i}\right)=0 \forall p_{i} \in P_{R} \cap S$ implies that $M_{k}\left(p_{i}\right)=0$ for any of $p_{i} \in I\left(t_{j}\right)$, for each $t_{j} \in T_{O P}$ representing the beginning of operations at $M_{k}$. Therefore, siphon is deadly marked at $M_{k}$ and PPNR is not live, by contradiction, there does not exits a siphon $S$ which holds the condition given in the statement of the theorem.

Conversely, for every siphon in PPNR, $\forall M_{k} \in R\left(M_{0}\right)$ : $M_{k}\left(p_{i}\right) \neq 0 \forall p_{i} \in P_{R} \cap S$. This implies that resource places in siphons remain marked for each reachable marking in PPNR, every siphon would not eventually become empty, which directly follows that every siphon has a marked trap. Hence PPNR is live.

Theorem 13: An PPNR is reversible if and only if it is live.

The statement of Theorem 13 is straightforward, for the characterization point of view for PPNR, liveness is similar to the reversibility.

The main requirement for PPNR is that every parallel processing flow in the system is able to complete, without any deadlock. The statements of the Theorems 10 to 13 depict the conditions under which this requirement is accomplished.

\section{APPLICATION EXAMPLE}

This section illustrates the PN model of parallel manufacturing processes with shared resources in the form of PPNR.

The manufacturing system given in this example produces the final product from three primitive parts by using four machining centers, two assembly stations $A_{1}$ and $A_{2}$, two robots $R_{1}$ and $R_{2}$ and a buffer $B$. Each machining centre $M C_{i}$ contains a machine $M_{i}, i=1,2,3,4$. When either $A_{1}$ or $A_{2}$ is ready to execute the assembly task, it requests both robots $R_{1}$ and $R_{2}$ and acquires them if they are available. When $A_{1}\left(A_{2}\right)$ completes the assembly task, it releases both the robots.

It is assumed that input parts are always available to be fixture and that the finished product to be removed. Further, once the system is executed, it can not be interrupted and 
system can not begin a new iteration before termination.

The production plan is given as follows:

1) Part 1 is machined by $M_{1}$ and part 2 is machined by $M_{2}$. Each part automatically fixtures to the pallet and loaded into a machine.

2) After processing, parts 1 and 2 enter the assembly station $A_{1}$ for producing part $S$.

3) Part 3 is machined first by $M_{3}$ and then by $M_{4} . \operatorname{In} M_{3}$, part 3 automatically fixtures to the pallet and loaded into a machine. After processing, the robot $R_{1}$ unloads the intermediate part from $M_{3}$ into the buffer $B$ and $M_{3}$ is released.

4) From the buffer $B$, intermediate part is automatically loaded into $M_{4}$ and processed. When $M_{4}$ finishes processing a part, the robot $R_{1}$ unloads a product, a part $T$, and $M_{4}$ is released.

5) The assembly station $A_{2}$ assembles parts $S$ and $T$ to produce the final product.

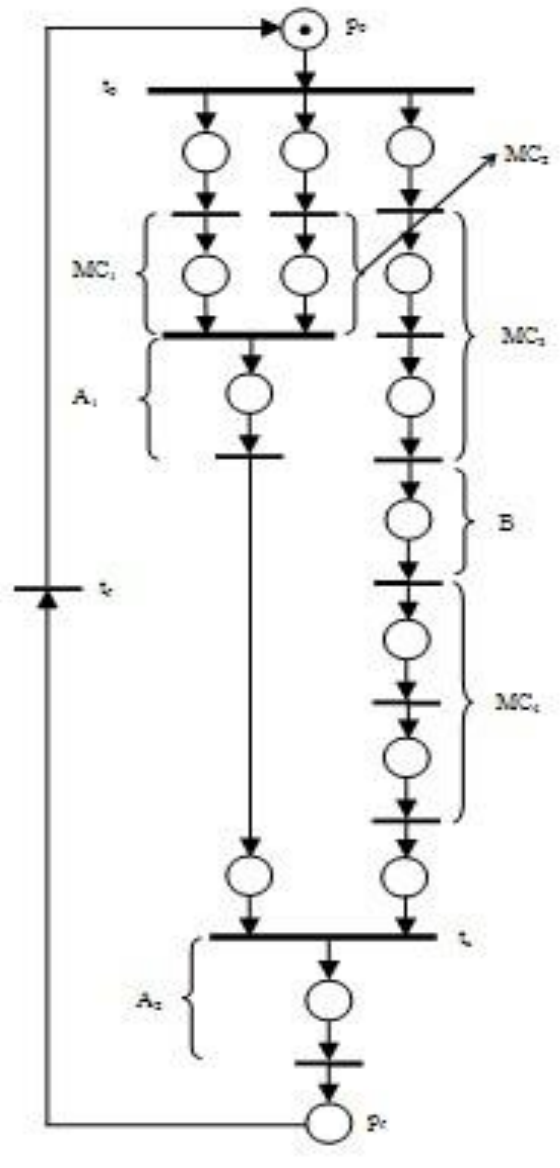

(a)

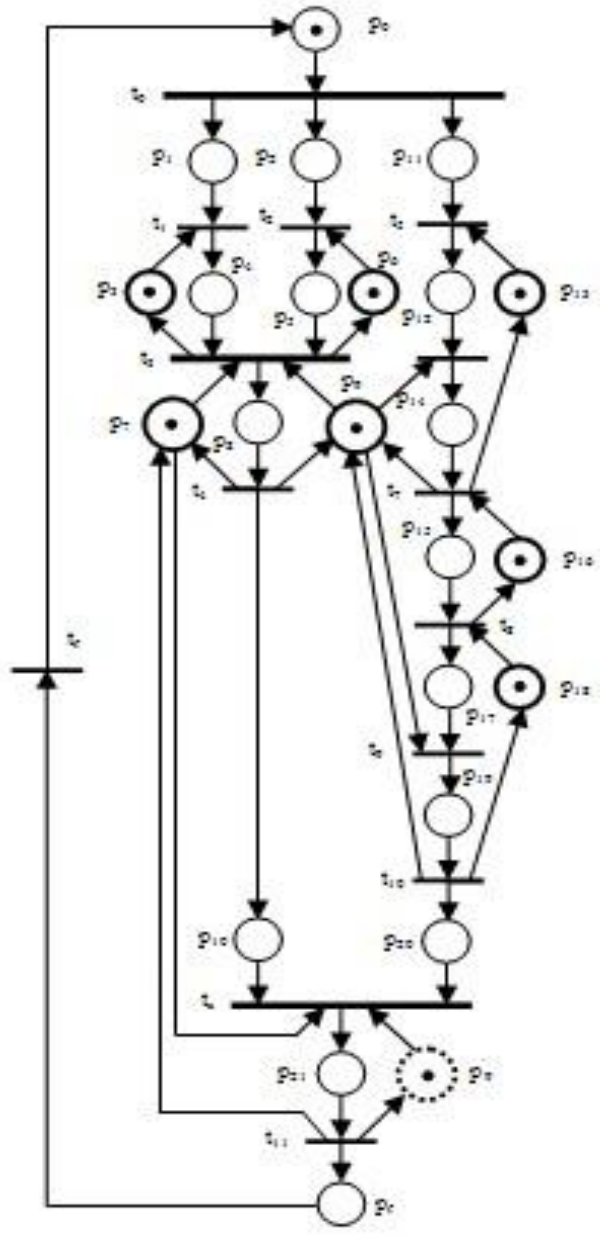

(b)

Fig. 2 (a) PPN and (b) PPNR of given manufacturing system

Modeling PPNR for given manufacturing system:

According to the explanation given in Section 3, firstly the PPN of production plan given above is constructed to indicate the process flow of each part type. Fig. 2(a) clearly depicts the parallel processing flows in manufacturing system as well as resource requirement for operations to be performed.

The PPNR is constructed in second step by adding the resource places denoting the availability of resources and resources shared by several processes. Fig. 2(b) shows the resultant PPNR and its transitions denote starting or finishing of the operations. The places of PPNR in Fig. 2(b) represent the operations performed in the manufacturing system and resource types which are explained in Table 1 . The shared robots $R_{1}$ and $R_{2}$ are represented by places drawn in Fig. 2(b) by larger circles while the robot $R_{1}$ is drawn twice and represented by the places $p_{9}$ and $p_{9}^{\prime}$ for clear presentation. In fact, both the places $p_{9}$ and $p_{9}^{\prime}$ are representation of single place.

From Property 9, every resource place has a marked minimal siphon. Further, For PPNR given in Fig. 2(b), every siphon contains a marked trap and would never become empty. Theorem 12 directly follows that given PPNR is live, which implies its reversibility. 


\section{CONCLUSION}

A new class of nets, PPNR, is presented to model the parallel processing of multiple parts on shared resources. The proposed PPNR has the capability to model the real-life manufacturing systems having complex resource sharing. It has been presented that the more complex production plans of manufacturing systems implicated in resource-sharing can be modeled by PPNR.
This paper further presents a number of characterizations of live and reversible PPNR based on siphons and minimal marked siphons. Simple structure based conditions and properties for conservative PPNR are presented for checking the liveness and reversibility. These characteristics help to identify the requirements of the structure of PPNR and its behavior. Furthermore, simple structural based conditions and properties, presented in this paper, are found practical for the synthesis of live and reversible PPNR.

Table 1: Interpretation of places of Fig. 2(b)

\begin{tabular}{|c|c|c|c|}
\hline $\begin{array}{l}\text { Operation } \\
\text { places }\end{array}$ & Interpretation & $\begin{array}{c}\text { Resource } \\
\text { places }\end{array}$ & Interpretation \\
\hline $\mathrm{p}_{0}$ & Initial place of PPNR & $\mathrm{p}_{3}$ & Machine $\mathrm{M}_{1}$ available \\
\hline $\mathrm{p}_{1}$ & Part 1 is available & $\mathrm{p}_{6}$ & Machine $\mathrm{M}_{2}$ available \\
\hline $\mathrm{p}_{2}$ & Part 2 is available & $\mathrm{p}_{7}$ & Robot $R_{1}$ available \\
\hline $\mathrm{p}_{4}$ & Part 1 machined by $\mathrm{M}_{1}$ & $\mathrm{p}_{9}$ & Robot $R_{2}$ available \\
\hline $\mathrm{p}_{5}$ & Part 2 machined by $\mathrm{M}_{2}$ & $\mathrm{p}_{13}$ & Machine $\mathrm{M}_{3}$ available \\
\hline $\mathrm{p}_{8}$ & Parts $1 \& 2$ assembled on $\mathrm{A}_{1}$ & $\mathrm{p}_{16}$ & Buffer B available \\
\hline $\mathrm{p}_{10}$ & Part $\mathrm{S}$ is available & $\mathrm{p}_{18}$ & Machine $\mathrm{M}_{4}$ available \\
\hline $\mathrm{p}_{11}$ & Part 3 is available & & \\
\hline $\mathrm{p}_{12}$ & Part 3 machined by $\mathrm{M}_{3}$ & & \\
\hline $\mathrm{p}_{14}$ & Intermediate part unloaded by $\mathrm{R}_{1}$ & & \\
\hline $\mathrm{p}_{15}$ & Intermediate part in buffer B & & \\
\hline$p_{17}$ & Intermediate part machined by $\mathrm{M}_{4}$ & & \\
\hline $\mathrm{p}_{19}$ & Intermediate part unloaded by $\mathrm{R}_{1}$ & & \\
\hline $\mathrm{p}_{20}$ & Part $\mathrm{T}$ is available & & \\
\hline $\mathrm{p}_{\mathrm{f}}$ & Final place of PPNR & & \\
\hline
\end{tabular}

\section{ACKNOWLEDGMENT}

First author (F. A.) is grateful to the Higher Education Commission of Pakistan and The Punjab Education Department, Pakistan for providing him a scholarship for pursuing doctoral studies at Harbin Institute of Technology Shenzhen Graduate School, Shenzhen (China).

\section{REFERENCES}

[1] M. Kamath, and N. Viswanadham, "Application of Petri net based models in the modeling and analysis of flexible manufacturing systems," Proceeding of IEEE International Conference on Robotics and Automation, vol. 3, 1986, pp. 312-317.

[2] J. Ezpeleta, , and J. Martinez, "Synthesis of live models for a class of FMS," Proceeding of IEEE International Conference on Robotics and Automation, vol. 3, 1993, pp. 557-563.

[3] M. C. Zhou, F. DiCesare, and A. A. Desrochers, "A hybrid methodology for synthesis of Petri net models for manufacturing systems," IEEE Trans on Robotics and Automation, 8(3), 1992, pp. 350-361.

[4] M. C. Zhou, K. McDermott and P. A. Patel, "Petri net synthesis and analysis of a flexible manufacturing system cell," IEEE Trans. on Systems, Man, and Cybernetics, 23(2), 1993, pp. 523-531.

[5] T. H. Sun, C. W. Cheng and L. C. Fu, "A Petri net based approach to modeling and scheduling for an FMS and a case Study," IEEE Trans. on Industrial Electronics, 41(6), 1994, pp. 593-601.

[6] M. C. Zhou, and K. Venkatesh, Modeling, Simulation and Control of FMS, a Petri net Approach. 1999, Word Scientific Publishing Co.

[7] H. Huang, Enhancing the property preserving Petri net process algebra for component-based system design (with application to designing multi-agent systems and manufacturing systems). 2004, PhD Thesis, City University of Hong Kong.

[8] K. H. Lee, J. Favrel and P. Baptiste, "Generalized Petri net reduction method," IEEE Trans. on Systems, Man, and Cybernetics, 17(2), 1987, pp. 297-303.

[9] M. C. Zhou, and F. DiCesare, "Parallel and sequential mutual exclusions for Petri net modeling for manufacturing systems with shared resources," IEEE Trans. on Robotics and Automation, vol. 7, 1991, pp. 515-527.

[10] N. Hamerrlian, "Refinement of open protocols for modeling and analysis of complex interactions in multi-agent systems," Lecture Notes in Artificial Intelligence, vol. 2691, 2003, pp. 423-434.
[11] Y. Souissi, "On liveness preservation by composition of nets via a set of places,” LNCS, Springer-Verlag, vol. 524, 1990, pp. 277-295.

[12] J. Ezpeleta, M. J. Colom and J. Martinez, "A Petri net based deadlock prevention policy for flexible manufacturing systems," IEEE Trans. on Robotics and Automation, vol. 11, 1995, pp. 173-184.

[13] H. Huang, L. Jiao and T. Y. Cheung, "Property-preserving composition of augmented marked graphs that share common resources," Proceeding of IEEE International Conference on Robotics and Automation, vol. 1, 2003, pp. 1446-1451.

[14] F. Chu, and X. L. Xie, "Deadlock analysis of Petri nets using siphons and mathematical programming," IEEE Trans. on Robotics and Automation, 13(6), 1997, pp. 793-804.

[15] Z. Li, and M. C. Zhou, "Elementary siphons of Petri nets and their application to deadlock prevention in flexible manufacturing systems" IEEE Trans. on Systems, Man, and Cybernetics-Part A: Systems and Humans, 34(1), 2004, pp. 38-51.

[16] Z. Li, and N. Wei, "Deadlock control of flexible manufacturing systems via invariant-controlled elementary siphons of Petri nets," Int. J Adv. Manuf. Technol, Springer-Verlag, vol. 33, 2007, pp. 24-35.

[17] M. Jeng, X. Xie, and M. Y. Peng, "Process nets with resources for manufacturing modeling and their analysis," IEEE Trans. on Robotics and Automation, 18(6), 2002, pp. 875-889.

[18] Z. Li, and M. C. Zhou, "On siphon computation for deadlock control in a class of Petri nets," EEE Trans. on Systems, Man, and Cybernetics-Part A: Systems and Humans, 38(3), 2008, pp. 667-679.

[19] Z. Li, M. C. Zhou and M. Jeng, "A maximally permissive deadlock prevention policy for FMS based on Petri net siphon control and the theory of regions," IEEE Trans. on Automation Science and Engineering, 5(1), 2008, pp. 182-188.

[20] E. R. Boer, and T. Murata, "Generating basis siphons and traps of Petri nets using the sign incidence matrix," IEEE Trans. on Circuits and Systems-I: Fundamental Theory and Applications, 41(4), 1994, pp. 266-271.

[21] R. Cordone, L. Ferrarini and L. Piroddi, "Some results on the computation of minimal siphons in Petri nets," Proceedings of the 42nd IEEE Conference on Decision and Control, 2003, pp. 3754-3759.

[22] A. Valmari, "The state explosion problem," Lectures on Petri Nets I: Basic Models, LNCS vol. 1491, 1998, pp. 429-528.

[23] T. Murata, "Petri nets: properties, analysis and application," In Proceedings of IEEE, 77(4), 1989, pp. 541-580.

[24] J. L. Peterson, Petri Net Theory and the Modeling of Systems, 1981, Prentice-Hall: Englewood Cliffs, NJ. 


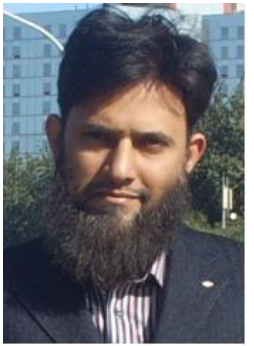

Education Department, Pakistan.

Farooq Ahmad received the M. Sc. degree from University of The Punjab and M. Phil. degree from GC University Lahore, Pakistan in Statistics in 1994 and 2001 respectively, and now he is $\mathrm{PhD}$ scholar in the Computer Science Department of Harbin Institute of Technology Shenzhen Graduate School, China. His research interests include the formal methods for system design, specifications and verification of the distributed and manufacturing systems using Petri nets.

$\mathrm{He}$ is also a Lecturer in Statistics in the Punjab

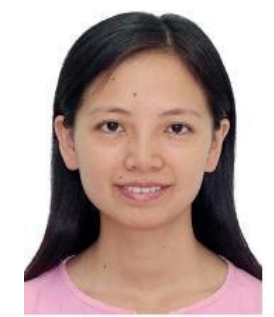

Hejiao Huang received the B. S. and M. S. degrees in Applied Mathematics form Shaan Xi Normal University in 1997 and 1999 respectively. Further, she received $\mathrm{PhD}$ degree in Computer Science from City University of Hong Kong in 2004. Her research interests include Petri net theory and applications, graph theory and application, optical and wireless mesh networks, machine learning and formal methods for system design.

Dr. Huang is currently an Associate Professor in the Department of Computer Science of Harbin Institute of Technology Shenzhen Graduate School, China.

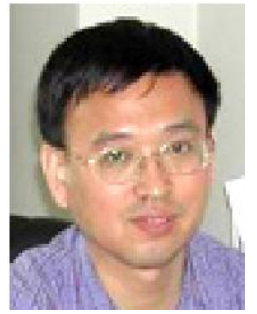

Xiao-long Wang received the B.E. degree in Computer Science from Harbin Institute of Technology, China, and the M.E. degree in Computer Architecture from Tianjin University, China, in 1982, and 1984, respectively, and the Ph.D. in computer science and engineering from Harbin Institute of Technology, China, in 1989, where he became an Associate Professor in 1990. He was a senior research fellow at the Polytechnic University from 1998 to 2000, a Professor of computer science at Harbin Institute of Technology, China. His research interests include artificial intelligence, machine learning, computational linguistics and Chinese information processing. 